\title{
CD90+ liver cancer cells modulate endothelial cell phenotype through the release of exosomes containing H19 IncRNA
}

\author{
Alice Conigliaro ${ }^{1 *+}$, Viviana Costa ${ }^{2 \dagger}$, Alessia Lo Dico ${ }^{3+}$, Laura Saieva ${ }^{3}$, Simona Buccheri ${ }^{3,4}$, Francesco Dieli ${ }^{3}$, \\ Mauro Manno ${ }^{5}$, Samuele Raccosta ${ }^{5}$, Carmine Mancone ${ }^{1,6}$, Marco Tripodi ${ }^{6,7}$, Giacomo De Leo ${ }^{3}$ \\ and Riccardo Alessandro $3,8^{*}$
}

\begin{abstract}
Background: CD90+ liver cancer cells have been described as cancer stem-cell-like (CSC), displaying aggressive and metastatic phenotype. Using two different in vitro models, already described as CD90+ liver cancer stem cells, our aim was to study their interaction with endothelial cells mediated by the release of exosomes.

Methods: Exosomes were isolated and characterized from both liver CD90+ cells and hepatoma cell lines. Endothelial cells were treated with exosomes, as well as transfected with a plasmid containing the full length sequence of the long non-coding RNA (IncRNA) H19. Molecular and functional analyses were done to characterize the endothelial phenotype after treatments.

Results: Exosomes released by CD90+ cancer cells, but not by parental hepatoma cells, modulated endothelial cells, promoting angiogenic phenotype and cell-to-cell adhesion. LncRNA profiling revealed that CD90+ cells were enriched in IncRNA H19, and released this through exosomes. Experiments of gain and loss of function of H19 showed that this LncRNA plays an important role in the exosome-mediated phenotype of endothelial cells.

Conclusions: Our data indicate a new exosome-mediated mechanism by which CSC-like CD90+ cells could influence their tumor microenvironment by promoting angiogenesis. Moreover, we suggest the IncRNA H19 as a putative therapeutic target in hepatocellular carcinoma.
\end{abstract}

Keywords: Exosomes, Long-non-coding RNA H19, CD90+ liver cancer cells, Angiogenesis

\section{Background}

Hepatocellular carcinoma ( $\mathrm{HCC})$ is the third leading cause of cancer mortality worldwide [1]. Primary HCC lesions can be removed completely when detected at an early stage, but intrahepatic recurrence of HCC and extrahepatic metastasis are very frequent, giving rise to a poor prognosis for patients $[2,3]$. It is widely accepted that both differentiated hepatocytes and cells with progenitor characteristics, known as cancer stem cells (CSCs), can cause

\footnotetext{
*Correspondence: alice.conigliaro@uniroma1.it; riccardo.alessandro@unipa.it ${ }^{\dagger}$ Equal contributors

'Dipartimento di Biotecnologie Cellulari ed Ematologia, Sapienza University of Rome, c/o Policlinico Umberto I, V Clinica Medica Viale Regina Elena, Rome 324-00161, Italy

${ }^{3}$ Dipartimento di Biopatologia e Biotecnologie Mediche, University of Palermo, Via Divisi 83-90133, Palermo, Italy

Full list of author information is available at the end of the article
}

HCC [4-7]. Forty percent of HCCs are clonal, and potentially derived from progenitor/stem cells. Moreover, these cells have a critical role in the development and progression of HCC [8]. Liver CSCs have been isolated from primary HCC specimens and patients' sera as circulating cells, and from HCC cell lines by use of surface antigens [9-11]. CD90, the epithelial cell adhesion molecule (EpCAM) and CD133 have been found to recognize three distinct cell populations that differ from one another in features and behavior in determining cancer phenotypes [12].

CD90 (Thy-1) is a 25-37 kDa glycophosphatidylinositol (GPI)-anchored protein expressed by several cells such as T-cells, neurons, endothelial cells and fibroblasts. It is involved in cell-to-cell and cell-matrix interaction, apoptosis, adhesion, migration, fibrosis, and

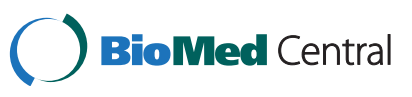


cancer development [13]. Concerning the liver, the expression of CD90 has been linked to hepatic stem/progenitor cells [14] and, during tumor growth, it has been correlated with an aggressive phenotype [15], and associated with low differentiated HCC and poor prognosis [16-18]. CD90+ CSCs obtained from HCC cell lines, from tumor tissues and peripheral blood as circulating cancer cells displayed, in contrast to the other CSC populations, a mesenchymal phenotype and, most importantly, a greater capacity to metastasize when injected into immunodeficient mice [11, 12, 19]. Moreover, recent data associate CD90 expression with early HCC recurrence [20]. Gene expression and miRNA analysis in CD90+ HepG2 cells have revealed an imbalance in the expression of apoptotic and anti-apoptotic genes compared with CD90 negative cells [21]. However, we are still far from understanding the molecular mechanisms underlying the more aggressive and metastatic phenotype of these cells compared with the other liver cancer cells.

Tumor development is dependent on the reciprocal interactions between cancer cells and the surrounding microenvironment. It is well known that in addition to pathways involving cell-to-cell contact and the release of soluble factors, cancer cells are able to communicate with the tumor microenvironment (e.g., myeloid cells, fibroblasts, endothelial cells) through the intercellular exchange of proteins and genetic materials via exosomes [22].

Exosomes are spherical membrane vesicles of endocytic origin, with an average size of 40 to $150 \mathrm{~nm}$ [23], released by both normal and diseased cells after the fusion of multivesicular bodies with the plasma membrane. First considered as collectors of cellular waste materials, exosomes have assumed a leading role in the regulation of the tumor microenvironment. Depending on their content, exosomes can affect tumor cells and surrounding stroma by influencing major cellular pathways, such as apoptosis, cell differentiation, angiogenesis, and metastasis [24]. These vesicles act as cargos that release bioactive molecules e.g., lipids, proteins, and nucleic acids in target cells. Interestingly, recent observations have identified a vesicle-mediated transfer of lncRNAs as an important mechanism in the development of HCC [25]. In this paper we demonstrate that CD90+ cells, derived from HCC cell lines, release exosomes that, in turn, are able to influence endothelial cells by promoting angiogenesis and stimulating their adhesive properties. Furthermore, our results suggest the lncRNA H19 as a possible mediator of angiogenic effects.

\section{Results}

CD90+ cells show a mesenchymal phenotype and actively release exosomes

As reported by Yang and colleagues [11], highly positive CSC-like CD90+ cells were isolated by cell sorting, starting from Huh7 cell line presenting a mean of $4 \%$ CD90+ cells and $2 \%$ CD90 high-expressing cells. After sorting, the purity of the selected $\mathrm{CD} 90+$ population was monitored during cell passages by FACS analysis, and the cells were kept in culture until they maintained a positivity for CD90 of over $90 \%$ (at approximately the $40^{\text {th }}$ passage). Isolated CD90+ cells, in contrast to the parental Huh7 and as already described by others [12], showed a mesenchymal phenotype, revealing a delocalized E-Cadherin and a lack of expression of HNF4 $\alpha$, a master regulator of hepatocytic differentiation (Fig. 1a). On the contrary, most of the cells were positive for vimentin, a component of intermediate filaments in mesenchymal cells (Fig. 1a). In order to evaluate the ability of Huh7 and its CD90+ subpopulation to release nanovesicles, the conditioned medium was collected, and the vesicles isolated as described by members of our group [26, 27]. Measures obtained by DLS revealed, in the ultracentrifuged cell culture medium, vesicles with an average size in diameter of $50 \mathrm{~nm}$ and $100 \mathrm{~nm}$ from CD90 + or Huh7 cell medium, respectively (Fig. 1b). This in line with the exosomes dimensions between 30 and $150 \mathrm{~nm}$ [28]. Moreover, Western blot analyses showed that Alix and Tsg101 markers are expressed but not enriched in exosomes released by CD90 + Huh7 (Fig. 1c).

\section{Exosomes released by CD90+Huh7 cells affect HUVECs by promoting tube formation and cell-cell adhesion}

CD90 + CSCs have been associated with metastasis and early recurrence in $\mathrm{HCC}[12,20]$. In order to evaluate whether the CD90+Huh7 cells were able to influence the tumor microenvironment, we treated HUVECs with exosomes released by CD90+ Huh7 cells or Huh7 parental cells (CD90 + exo and Huh7exo). Endothelial cells rapidly internalized exosomes from both cell types; uptake was evident after one-hour of incubation at $37{ }^{\circ} \mathrm{C}$, and increased over the course of six hours (Fig. 1d). Eighteen hours after exosome treatment, real-time PCR analysis revealed that the addition of $\mathrm{CD} 90+$ exo, but not of Huh7exo, highly increased the mRNA levels of the proangiogenic factor VEGF and its receptor VEGF-R1 in endothelial cells (Fig. 2a). ELISA assay showed that HUVECs treated with CD90 + exo released three-fold more VEGF (Fig. 2b, left panel). Moreover, a significant increase in the number and the length of tubular-like structures was observed when HUVECs were treated with CD90 + exo compared with Huh7exo (Fig. 2b, middle and right panels).

Liver CD90+ CSCs were found circulating in HCC patients and in metastatic colonies [19]. For this reason, we tested the ability of exosomes released by the hepatoma cell line or by sorted CD90+ cells to modulate the adhesion to an endothelial cell monolayer, a crucial event for intra- or extra-vasation. As revealed by real- 


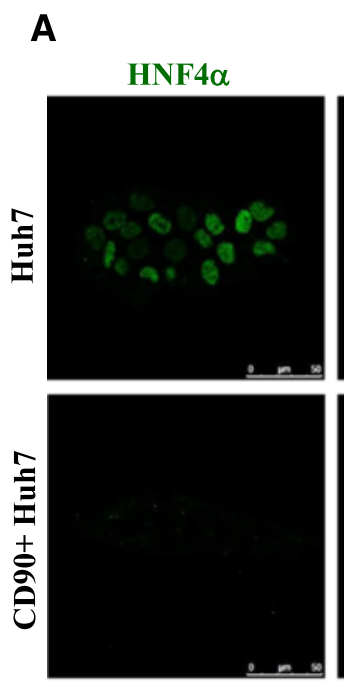

B

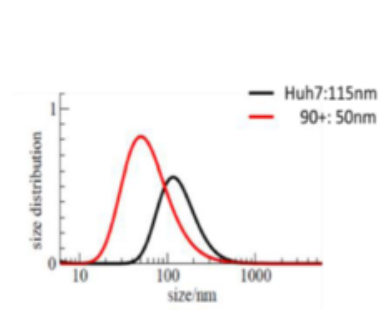

D

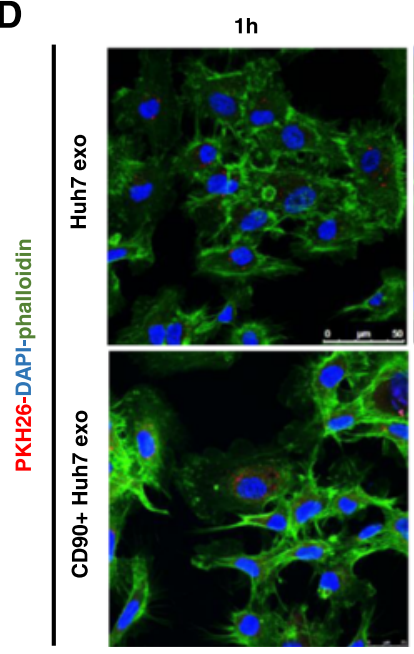

E-CAD
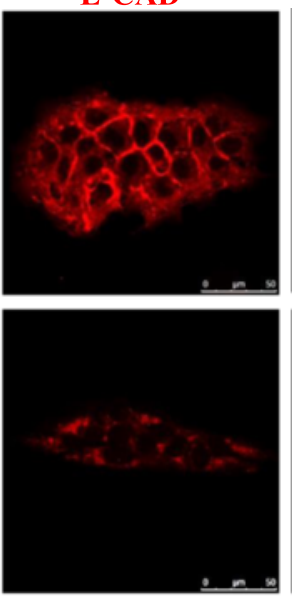

c

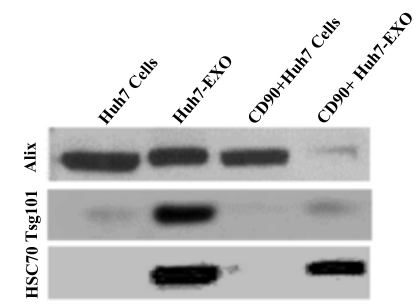

3h

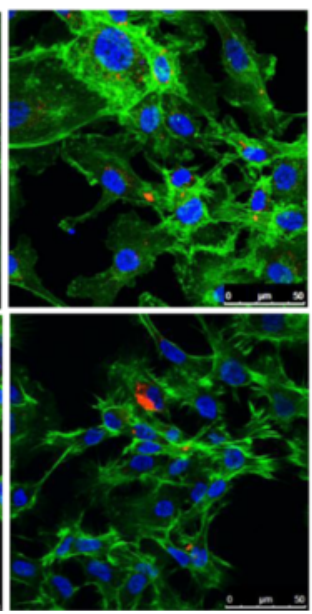

$6 h$

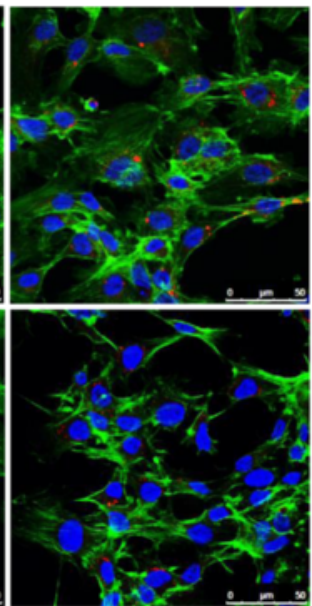

Fig. 1 CD90+ population. a Huh7 and sorted CD90+ Huh7 were stained for hepatocytic (HNF4alpha), epithelial (E-Cadherin) and mesenchymal (Vimentin) markers, in blue the nuclear staining with DAPI. Characterization of isolated exosomes. $\mathbf{b}$ Dynamic light scattering of vesicles isolated from Huh7 (in black) and from CD90 + Huh7 cells (in red). c Western blot for endosomal markers Alix, Tsg101 and HSC70 in Huh7 and CD90+Huh7 population with their relative exosomes. $\mathbf{d}$ Confocal microscopy analysis on HUVECs treated for 1, 3, and $6 \mathrm{~h}$ with $5 \mu \mathrm{g} / \mathrm{ml}$ of exosomes from CD90+ or Huh7 cells. HUVECs were stained with phalloidin Alexa Fluor488 (green), nuclear counterstaining was done using DAPI (blue), exosomes were labelled with PKH26 (red)

time PCR (Fig. 2a), and confirmed by FACS analysis (Fig. 2c), treatment of HUVECs with CD90 +-derived exosomes modulated intercellular adhesion molecules, inducing an increase in the expression of ICAM-1. No significant differences were found in VCAM and VE-Cadherin gene expression (data not shown). To validate our data, we did an adhesion assay, pretreating endothelial cells with exosomes. As shown in Fig. 2d, CD90 + exo caused a two-fold increase in adhering cells compared with pre-treatment Huh7 exo.

To further confirm our observation, the same experiments were performed with SkHep, a hepatoma cell line 


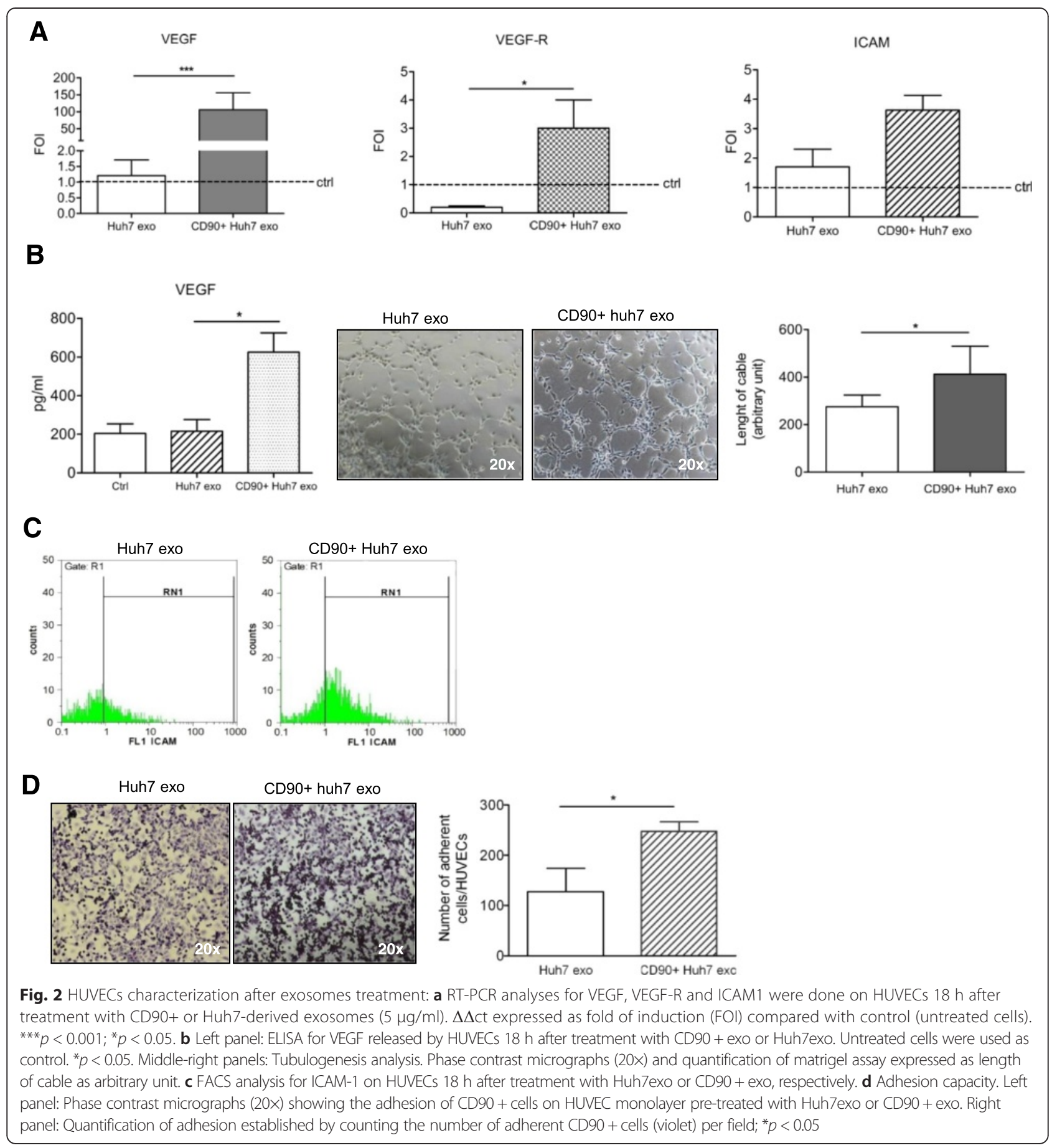

already characterized as $100 \% \mathrm{CD} 90+$, and displaying mesenchymal stem cell characteristics [29]. Additional file 1 illustrates the characterization of exosomes released by SkHep and their uptake by HUVECs, that present different features compared to CD90+ Huh7 derived exosomes. In addition, measures obtained by DLS revealed, in the ultracentrifuged SKHep culture medium, exosomes with an average size in diameter of $70 \mathrm{~nm}$ expressing high level of the exosomal markers
TSG101 and HSC70. As observed for CD90 + exo, the SkHep-derived exosomes induced a pro-angiogenic stimulus in endothelial cells, modifying their transcriptional profile and enhancing tube formation in matrigel, as well as increasing the adhesive properties of HUVECs.

In summary, our results showed that exosomes released by CSC-like CD90+ liver cells, but not from hepatoma cells, induce pro-angiogenic stimuli in HUVECs, 
and influence the adhesion between CD90+ cells and endothelial cells.

\section{CD90+ cells express the IncRNA H19 and release it via exosomes}

It has been confirmed that dysregulation of lncRNAs is associated with several human tumors and, recently, a contribution of lncRNAs to hepatocarcinogenesis was found [30-32]. In order to clarify the molecular mechanism driving the modifications induced in HUVECs by CD90 + -derived exosomes we did an lncRNA profile study in CD90+ cells and parental Huh7 by analyzing the expression of 90 different lncRNAs. In Fig. 3a (left and middle panel), the RNAs over-expressed in CD90+Huh7 cells compared with Huh7 parental cells with at least a ten-fold increase are listed. Among these, Air, Hotair, LincRNAROR, Hulc, and H19 have already been identified as positively correlated with hepatocellular carcinoma [31, 33, 34]. We focused our interest on H19, expression of which has been previously associated with metastasis $[35,36]$. In line with recent articles, which have demonstrated that hepatocellular carcinoma cells release exosomes containing lncRNA [25, 37], we investigated the expression in exosomes, of those LncRNAs that we found overexpressed in cells. As shown in Fig. 3a right panel the LncProfiler performed on CD90+ Huh7 and Huh7-derived exosomes evidences that $\mathrm{H} 19$ was 10 -fold up-regulated in exosomes derived from CD90+ Huh7, compared to parental cell line. The Real-time PCR confirmed that vesicles released by CD90 + cells (both sorted or SkHep cells) are highly

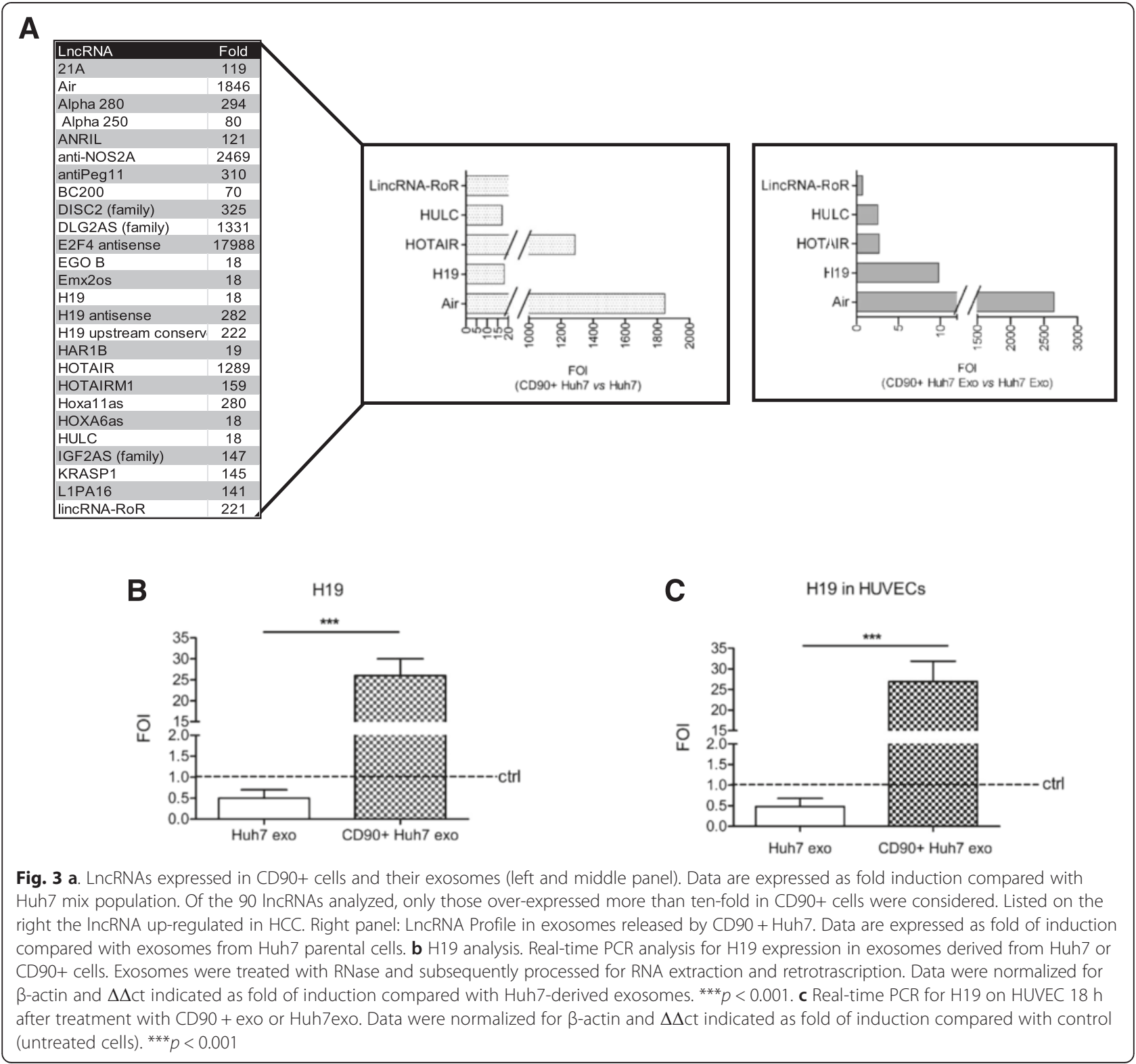


enriched in H19 transcript compared with vesicles from Huh7 parental cells (Fig. 3b, S1e). Moreover, treatment with CD90 +-derived exosomes induced in HUVECs an increase in H19 transcript (Fig. 3c, S1f). These data suggest a transport of H19 lncRNA from CD90+ cells to HUVECs, even if we cannot exclude a stimulation of endogenous lncRNA.

\section{LncRNA H19 stimulates angiogenesis and promotes the adhesion of CD90+Huh7 cells to endothelial cell monolayer}

To investigate a possible role of $\mathrm{H} 19$ as mediator of proangiogenic and adhesive stimuli in HUVECs, we transfected endothelial cells with the entire sequence of the lncRNA H19 (pH19). As shown in Fig. 4a, H19 overexpression in HUVECs induced a transcriptional modulation similar to that obtained after CD90 + exo treatment. Real-time PCR indicated that the over expression of H19 induced a significant increase in the VEGF and ICAM1 transcripts, while, no modulation compared to controls was observed for the transcription of VEGF-R1, VCAM and VE-cadherin (Fig. 4a right panel). The ELISA assay (Fig. 4a left panel) found, for the first time to our knowledge, a substantial increase in VEGF release induced by lncH19. Moreover, a rise in the number and length of tubes was found in HUVECs transfected with pH19 (Fig. 4b), while FACS analysis (Fig. 4c) indicated an increase in the number of ICAM-1-expressing cells induced by $\mathrm{H} 19$ overexpression, thus explaining the more adhesive phenotype of HUVECs. The adhesion assay, in fact, revealed a two-fold increase in adhering CD90+ cells when HUVECs were transfected with pH19 (Fig. 4d).

Overall, these data demonstrate, for the first time to our knowledge, the ability of the lncRNA H19 to stimulate angiogenesis, and to favor cell-cell interaction, allowing us to postulate $\mathrm{H} 19$ as a possible mediator of pro-metastatic properties of exosomes released by CD90+ cells. To confirm our hypothesis, $\operatorname{lncH} 19$ was silenced in HUVECs concomitantly with CD90 + exo treatment. As shown in Fig. 5a, the silencing of H19 abrogated the exosome-mediated induction of VEGFR1, while no modulation was revealed in the expression of ICAM1. Concerning the VEGF, even if the reduction of transcript did not appear significant (5a), the release of VEGF protein induced by exosome treatment was totally inhibited by H19 silencing (5b).

\section{Discussion}

CD90+ liver CSCs have been found in primary tumors, and circulating in the blood of HCC patients, and are associated with early recurrence, metastasis, and poor prognosis $[19,20]$. Our study highlights the ability of CSC-like CD90+ cells, but not hepatoma cells, to influence endothelial cell phenotype through the release of exosomes.

In a solid tumor, the CSC's niche is composed of an extracellular matrix (ECM), mesenchymal stem cells, tumoral cells, immune cells, and endothelial cells, all of which converge in determining the fate of CSCs through extracellular signals [38]. Little is known about the modulation of the tumor microenvironment by CSCs. Several studies have described exosomes as signaling extracellular organelles that modulate the tumor microenvironment, promoting angiogenesis and tumor progression [27, 39]. Our data indicate that exosomes released by CSC-like CD90+ liver cells are able to promote an angiogenic phenotype in cultured endothelial cells. CD90 +-derived exosomes induced in HUVECs an increase in the production and secretion of VEGF, the most powerful pro-angiogenic cytokine, as well as of its receptor VEGF-R1. This increase was accompanied by an amplification in the number and length of tube-like structures formed by HUVECs in culture.

It is abundantly documented that metastatic processes induce changes in the endothelial surface antigens, with an increase in adhesion molecules, which, in turn, favor the adhesion and the consequent intra- or extra-vasation of metastatic cells. We found that exosomes released by CD90+Huh7 cells, and not by hepatoma cells, increased the number of HUVECs expressing ICAM-1 and, more extensively, increased the adhesion between endothelial cells and the CSC-like CD90+ cells. Our data also indicate that the $\mathrm{CD} 90+$ released exosomes may be able to promote metastasis.

Recently, Patel et al. demonstrated that lncRNA could be selectively packaged in extracellular vesicles released by hepatoma cell lines and transported to other cells, with subsequent modulation of cellular function [25, 40]. LncRNA are emerging as molecular players in several biological processes acting at epigenetic, transcriptional and post-transcriptional levels or processing small non-coding RNAs [41]. H19 was among the first lncRNAs to be identified and studied principally for its monallelic expression, and as regulator of IGF2 abundance $[42,43]$. As already described for other IncRNAs, H19 can work as a microRNA sponge, miRNAs precursor, or epigenetic modulator [44, 45], and has been found overexpressed in several tumors, and able to promote tumor growth $[46,47]$ and progression $[47,35,36]$. Concerning the liver, H19 has been clearly involved in hepatocarcinogenesis [48] and hepatic metastases [49]. Several indications correlate H19 with angiogenesis $[50,51]$. Northern analysis has indicated a high expression of H19 during development of rat aorta that decreases in differentiated tissue and, interestingly, re-appears following vascular injury in vivo and in 

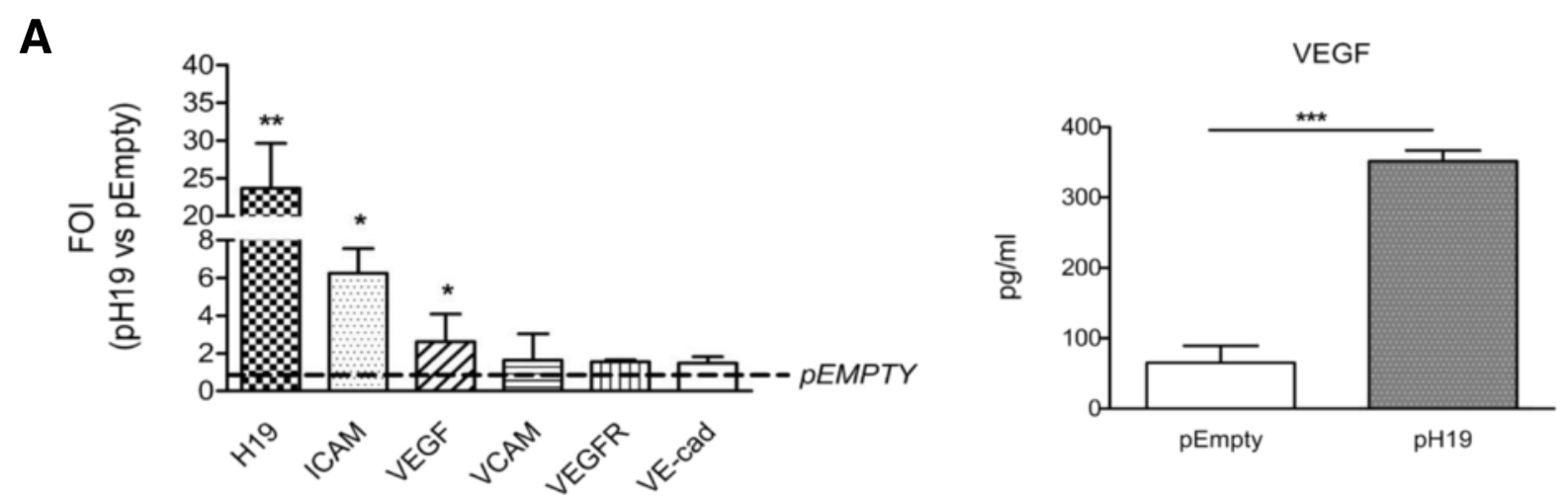

B
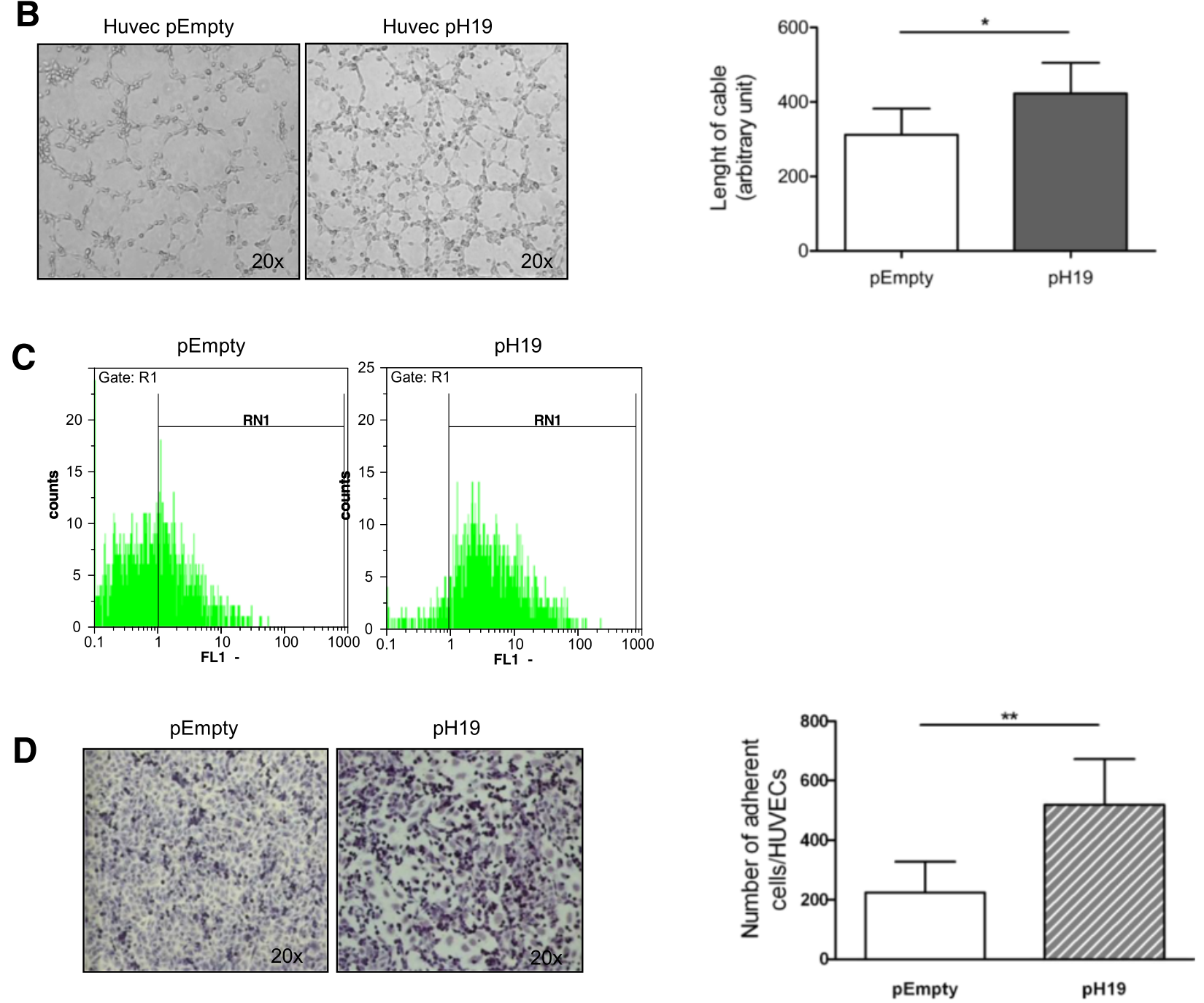

Fig. 4 H19 overexpression. a Left panel: Real-time PCR performed on HUVECs 18 h post-transfection. Data were normalized for $\beta$-actin and $\Delta \Delta \mathrm{ct}$ expressed as fold of induction pH19 vs. pEmpty ${ }^{* *} p<0.01$; ${ }^{*} p<0.05$. Right panel: ELISA assay for VEGF level in supernatant from HUVECs $18 \mathrm{~h}$ after transfection. ${ }^{* *} p<0.001$. b Left Panel: Phase contrast $(20 \times)$ of tubulogenesis assay performed $18 \mathrm{~h}$ after transfection. Right panel: quantification of matrigel assay expressed as length of cable as arbitrary unit* $p<0.05$. c FACS analysis for ICAM expression in HUVEC transfected cells. $\mathbf{d}$ Left Panel: Phase contrast micrographs (20X) showing the adhesion of CD90 + cells on HUVEC monolayer transfected with pEmpty or pH19. Right Panel Quantification was calculated by counting the number of adherent CD90+ cells (violet) per field. ${ }^{*} p<0.01$ 

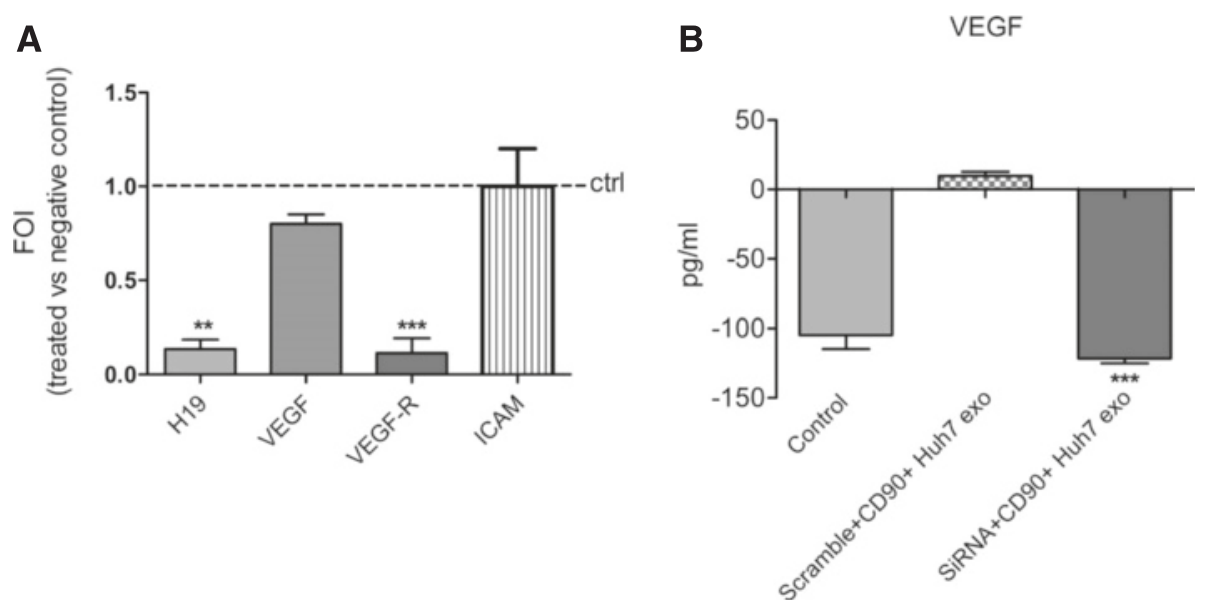

Fig. 5 a Real-time PCR for H19, VEGF, VEGFR1 and ICAM1 from HUVECs transfected with H19 siRNA or negative scramble and treated with CD90 + exo. Data were normalized for $\beta$-actin and $\Delta \Delta$ ct expressed as fold of induction siRNA H19 versus negative control. ${ }^{* *} p<0.01$, ${ }^{* * *} p<0.001$ b ELISA assay for VEGF detection on the supernatant from HUVECs treated as indicated above. ${ }^{* *} p<0.001$

vitro [50], though no observations of the overexpression of H19 in endothelial cells have been published.

In this study, we demonstrate, for the first time to our knowledge, that H19 is highly expressed in a subpopulation of hepatoma cells that expose the surface antigen CD90 and are characterized, by others, as CSC-like cells $[11,12,15,29]$. We found that CD90+Huh7 cells package lncRNA H19 inside exosomes, thus delivering it to possible target cells. Exosomes released by CD90+ liver cancer cells could be internalized by endothelial cells, influencing these in a pro-metastatic way. Moreover, we identified in $\mathrm{H} 19$ an important player of this process. $\mathrm{H} 19$ overexpression in endothelial cells is able to upregulate the VEGF production and release, increase the ability of HUVEC cells to arrange in vitro tubular-like structures, and promote heterotypic adhesion between endothelial cells and CSC-like liver cells. Silencing experiments revealed LncRNAH19 as the principal player of the exosome-mediated VEGF increase, while suggested the presence of other molecular actors that, transported or induced by CD90 +-derived exosomes, and together with $\mathrm{H} 19$, affect endothelial cells in a prometastatic way. However, the mechanisms of action through which this lncRNA controls an endothelial phenotype remain to be elucidated.

\section{Conclusion}

Our in vitro experiments demonstrated that CD90+ liver cancer cells release exosomes that, in turn, are able to affect endothelial cells in a pro-metastatic way. Exosomes derived by CD90+Huh7 cells and H19 may represent two new therapeutic targets for reducing recurrence and metastasis of $\mathrm{HCC}$.

\section{Material and methods Cell culture and reagents}

Human umbilical vein endothelial cells (HUVECs) were obtained from Lonza (Verviers, Belgium) and grown in endothelial growth medium (EGM, bullet kit, Lonza) according to supplier's instructions. Huh7 cells and SkHep cells were cultured in DMEM medium (Euroclone, UK), and supplemented with $10 \%$ fetal bovine serum (Euroclone, UK), 2 mM L-glutamine, $100 \mathrm{U} / \mathrm{ml}$ penicillin and $100 \mathrm{mg} / \mathrm{ml}$ streptomycin (Euroclone, UK).

\section{Sorting CD90+Huh7 cells}

Huh-7 human hepatocellular carcinoma cells were stained with anti-CD90 PE (BD Pharmingen ${ }^{\text {Tм }}$ 555596), and surface marker was determined by flow cytometry. CD90+ and CD90- cells were sorted through a FACSAria I (BD Biosciences). A purity check was done after the sorting by re-running a small fraction of the sorted populations. All cells showed over $85 \%$ purity.

\section{Immunocytochemistry}

Immunocytochemistry was done on PFA $4 \%$ fixed cells, and stained with the following antibodies: the primary antibodies were anti-E-Cadherin (BD Biosciences 610181), anti-HNF4a (Abcam ab41898), and antiVimentin (Epitomics, 2707-1); the secondary antibodies were Alexa-Fluor 488 and Alexa-Fluor 594, from Molecular Probes. The nuclei were stained with NucRed ${ }^{\odot}$ Live 647 (Catalog number: R37106, Life Technologies), and preparations were analyzed by confocal microscopy (Leica TSC SP8). 


\section{Exosome preparation and characterization}

Huh7, CD90+ Huh7 and Sk-Hep cells were grown with $10 \%$ ultracentrifugated FBS, and conditioned medium was collected $48 \mathrm{~h}$ after culture; exosomes were subsequently isolated by serial centrifugation [26]. Briefly, culture medium was centrifuged subsequently for $5 \mathrm{~min}$ at $300 \times \mathrm{g}, 15 \mathrm{~min}$ at $3,000 \times \mathrm{g}, 30 \mathrm{~min}$ at $10,000 \times \mathrm{g}$ and ultracentrifuged $90 \mathrm{~min}$ at $100,000 \times \mathrm{g}$ in a Type $70 \mathrm{Ti}$, fixed angle rotor. Peletted exosomes were washed and then resuspended in PBS. Exosome protein content was determined with the Bradford assay (Pierce, Rockford, IL, USA). On average we recovered 10 micrograms of vesicles from $25 \mathrm{ml}$ of conditioned medium from $3 \times 10^{6}$ cells. The intensity autocorrelation functions of diluted vesicle samples were measured by dynamic light scattering (DLS) using a Brookhaven Instruments BI-9000 correlator and a BI200-SM goniometer, equipped with a solid-state laser tuned at $532 \mathrm{~nm}$. The size distribution was determined from the vesicle diffusion coefficients by standard analysis [52]. Thirty $\mu \mathrm{g}$ of protein for each sample, exosomes, and cells, were analyzed by western blot for Alix (3A9-Cell Signaling Technology \#2171S),) Tsg101 (Santa Cruz Biotechnology sc-7964) and HSC70 (Santa Cruz Biotechnology sc-7298).

\section{Uptake of exosomes by HUVECs}

Exosomes from Huh7, CD90+ Huh7 and SkHep cells were labeled with PKH26 according to supplier's instructions, suspended in low serum medium $(5 \mu \mathrm{g} / \mathrm{ml})$, and incubated with HUVECs for 1,3 , and $6 \mathrm{~h}$ at $4^{\circ}$ or $37^{\circ} \mathrm{C}$. After incubation, cells were processed as previously described [26].

\section{HUVECs treatment}

HUVECs were grown at a density of 100.000cells/well in a 12 wells plate, and treated for $18 \mathrm{~h}$ with $5 \mu \mathrm{g} / \mathrm{ml}$ of exosomes in low serum medium; untreated cells were considered control. Plasmid for psiCHECK2-H19 and the Empty vector psiCHECK2 (kindly provided by $\mathrm{Dr}$ Y. Huang [45]]), H19 siRNA (SR319206B Origene Technologies) and scramble negative control (SR30004 Origene Technologies) were transfected in HUVECs with Attractene Transfection Reagent (cat.number.1051531, Quiagen) following manufacturer's indications.

\section{RNA extraction and real-time PCR}

RNA was extracted using the commercially available illustra RNAspin Mini Isolation Kit (GE Healthcare), according to manufacturer's instructions. Total RNA was reverse-transcribed to cDNA using the High Capacity cDNA Reverse Transcription Kit (Applied Biosystem). RT-QPCR was done in 48-well plates using the StepOne Real-Time PCR system (Applied Biosystem). Realtime PCR was performed in duplicates for each data point. For sybr-green method the oligonucleotide used were $\beta$-actin for 5 -ATCAAGATCATTGCTCCTCCTGA3'rev 5'CTGCTTGCTGATCCACATCTG-3'; H19 for5'GCACCTTGGACATCTGGAGT-3'rev5'-TTCTTTCCAG CCCTAGCTCA-3', VEGF for5'-CGAGGGCCTGGAGTG TGT-3'rev5'-CGCATAATCTGCATGGTGATG-3', VEGFR1 for5'-CGGTCAACAAAGTCGGGAGA-3'rev5'-CAG TGCACCACAAAGACACG-3', VE-CADHERIN for5'GATCAAGTCAAGCGTGAGTCG-3' rev5'-AGCCTCT CAATGGCGAACAC-3'. VCAM1, ICAM, H19 and $\beta$ actin transcript levels were measured by TaqMan RealTime PCR using the TaqMan gene expression assay: Hs00174239_m1, HS 00277001_m1, Hs00262142_g1 and Hs99999903_m1, respectively (Life Technologies,). Changes in the target mRNA content relative to housekeeping were determined with the $\Delta \Delta \mathrm{ct}$ Method.

\section{Endothelial tube formation assay}

HUVECs were seeded at 50,000 cells/well in growth factor-reduced Matrigel-coated 24 well plate and incubated up to $2 \mathrm{~h}$ at $37^{\circ} \mathrm{C}$. Tube formation was examined under an inverted microscope and photographed at $20 \times$ magnification. The length of the cables was measured manually with IMAGE-J software (http://rsbweb.nih.gov/ij/).

\section{FACS analysis}

Two hundred thousand $(200,000)$ cells were washed in PBS and incubated with $0.5 \mu \mathrm{g}$ ICAM-1-FITC (sc-107, Santa Cruz). Viable cells were gated by forward and side scatter, and analyzed on 100,000 acquired events for each sample. Samples were analyzed on a Partec CyFlow Space using the Partec FloMax ${ }^{\circ}$ software.

\section{Adhesion assay}

In order to evaluate the ability of CD90+ Huh7 cells and SkHep cells to adhere to HUVECs, an adhesion assay was performed, as previously described [26].

\section{ELISA}

HUVEC conditioned medium was collected $18 \mathrm{~h}$ after exosome treatment or transfection with pH19 or pEmpty. VEGF concentrations were quantified using the ELISA kit (KHG0111, LifeTechnologies), according to manufacturer's protocol.

\section{Array for long non-coding RNA}

In order to study lncRNA expressed in the sorted population, a LncProfiler lncRNA qPCR array was performed (System Bioscience) on Huh7, CD90 + Huh7 cells and their exosomes following manufacturer's indications. After amplification, $\Delta \Delta$ ct of CD90 + Huh7 was normalized on $\Delta \Delta$ ct of Huh7, and data were expressed as fold induction of the sorted population compared with the parental cells. 


\section{Statistical analysis}

In vitro experiments were repeated three times, giving reproducible results. Data are presented as mean values \pm standard deviation (SD) of three independent experiments. Statistical analysis was done using Prism 4 (GraphPad Software Inc., San Diego, CA, USA); one-way ANOVA (non-parametric) was performed, followed by Dunnett's multiple comparison test.

\section{Additional file}

\section{Additional file 1: (a) Characterization of isolated exosomes.}

Left panel: DSL for exosomes released by SKHep Middle panel: Western blot forTsg101 and HSC70 in SkHep cells and their relative exosomes. Right panel: Confocal microscopy analysis on HUVECs treated for 1, 3 and 6 hours with $5 \mathrm{mg} / \mathrm{ml}$ of SKHep-derived exosomes. HUVECs were stained with phalloidin Alexa Fluor (green), nuclear counterstaining was performed using DAPI (blue), exosomes were labelled with PKH26 (red). (b) Target analysis. Real time-PCR analysis on HUVECs treated for $18 \mathrm{~h}$ with $5 \mathrm{mg} / \mathrm{ml}$ of SkHep-derived exosomes. Normalized for b-actin the DDct were indicated as fold of induction respect to control (untreated cells). ${ }^{*} p<0.05$. (c) Tubulogenesis of HUVECs after exosomes treatment. Matrigel assay performed on HUVECs cells after 18 hour of $5 \mathrm{mg} / \mathrm{ml}$ SkHep-derived exosomes. Left panel: phase contrast, magnification 20x. Right panel: quantification of matrigel assay expresses as length of cable as arbitrary unit ${ }^{* *} \mathrm{p}<0.01$. (d) Adhesion assay of SkHep cells on HUVECs. Left panel: phase contrast, magnification 10X. Right Panel: quantification of Huh7 or SKHep cells adherent on HUVECs, performed by counting the number of CD90+adherent cells (violet) per field ${ }^{* * *} \mathrm{p}<0.001$. (e) Analysis on $\mathrm{H} 19$ expression in exosomes. Real time-PCR analysis for $\mathrm{H} 19$ expression performed on SkHep-derived exosomes respect to Huh7 derived exosomes. Normalized for b-actin the DDct were indicated as fold of induction. ${ }^{* *} \mathrm{p}<0.01$ (f) Comparison of $\mathrm{H} 19$ expression in HUVECs after exosomes treatment. Real time-PCR analysis for H19 expression on HUVECs treated for $18 \mathrm{~h}$ with $5 \mathrm{mg} / \mathrm{ml}$ of SkHep or Huh7 exosomes. Normalized for b-actin the DDct were indicated as fold of induction respect to control (untreated cells). (DOCX $855 \mathrm{~kb}$ )

\section{Abbreviations}

IncRNA: Long-non-coding RNA; HCC: Hepatocellular carcinoma; HUVEC: Human umbilical vein endothelial cell; CSC: Cancer stem cell; VEGF: Vascular endothelial growth factor; ICAM-1: Intercellular adhesion molecule-1.

\section{Competing interests}

The authors declare that they have no competing interests.

\section{Authors' contributions}

$A C, R A, V C, A L D$ contributed to the conception and design of the study. AC, RA, VC, ALD, LS, SB, SR, MM, RA contributed to the generation, collection, assembly, analysis and/or interpretation of data. AC, RA, VC, ALD, MT, CM, FD, $\mathrm{GDL}$, contributed to drafting or revision of the manuscript. All authors approved the final version of the manuscript.

\section{Financial supports}

MIUR Ministero dell'Universita e Ricerca Scientifica (FIRB 2012- RBFR12NSCF_002); AIRC Associazione Italiana per la Ricerca sul Cancro (12763).

\section{Author details}

'Dipartimento di Biotecnologie Cellulari ed Ematologia, Sapienza University of Rome, c/o Policlinico Umberto I, V Clinica Medica Viale Regina Elena, Rome 324-00161, Italy. ${ }^{2}$ Laboratory of Tissue Engineering - Innovative Technology Platforms for Tissue Engineering (PON01-00829), Rizzoli Orthopedic Institute, Palermo, Italy. ${ }^{3}$ Dipartimento di Biopatologia e Biotecnologie Mediche, University of Palermo, Via Divisi 83-90133, Palermo, Italy. ${ }^{4}$ Servizio di Diabetologia, Dipartimento per la cura e lo studio della patologie addominali e dei trapianti addominali, ISMETT IRCCS, Palermo, Italy.
${ }^{5}$ Institute of Biophysics, National Research Council of Italy, Palermo, Italy. ${ }^{6}$ National Institute for Infectious Diseases L. Spallanzani, IRCCS, Rome, Italy. ${ }^{7}$ Istituto Pasteur-Fondazione Cenci Bolognetti, Dipartimento di Biotecnologie Cellulari ed Ematologia, Sapienza University of Rome, Rome, Italy. ${ }^{8}$ Institute of Biomedicine and Molecular Immunology (IBIM), National Research Council of Italy, Palermo, Italy.

Received: 19 May 2015 Accepted: 3 August 2015

Published online: 14 August 2015

\section{References}

1. Forner A, Llovet JM, Bruix J. Hepatocellular carcinoma. Lancet. 2012;379(9822):1245-55. doi:10.1016/50140-6736(11)61347-0S01406736(11)61347-0.

2. Uchino K, Tateishi R, Shiina S, Kanda M, Masuzaki R, Kondo Y, et al. Hepatocellular carcinoma with extrahepatic metastasis: clinical features and prognostic factors. Cancer. 2011;117(19):4475-83. doi:10.1002/cncr.25960.

3. Yoo DJ, Kim KM, Jin YJ, Shim JH, Ko GY, Yoon HK, et al. Clinical outcome of 251 patients with extrahepatic metastasis at initial diagnosis of hepatocellular carcinoma: does transarterial chemoembolization improve survival in these patients? J Gastroenterol Hepatol. 2011;26(1):145-54. doi:10.1111/j.1440-1746.2010.06341.x.

4. Roncalli M, Park YN, Di Tommaso L. Histopathological classification of hepatocellular carcinoma. Digestive and liver disease. Off J Italian Soc Gastroenterol Italian Assoc Study Liver. 2010;42 Suppl 3:S228-34. doi:10.1016/S1590-8658(10)60510-5.

5. Suzuki A, Zheng Y, Kondo R, Kusakabe M, Takada Y, Fukao K, et al. Flow-cytometric separation and enrichment of hepatic progenitor cells in the developing mouse liver. Hepatology. 2000;32(6):1230-9. doi:10.1053/jhep.2000.20349.

6. Oishi N, Yamashita T, Kaneko S. Molecular biology of liver cancer stem cells. Liver Cancer. 2014;3(2):71-84. doi:10.1159/000343863.

7. Yoon SK. The biology of cancer stem cells and its clinical implication in hepatocellular carcinoma. Gut Liver. 2012;6(1):29-40. doi:10.5009/gnl.2012.6.1.29

8. Yao Z, Mishra L. Cancer stem cells and hepatocellular carcinoma. Cancer Biol Therapy. 2009;8(18):1691-8.

9. Chiba T, Kita K, Zheng YW, Yokosuka O, Saisho H, Iwama A, et al. Side population purified from hepatocellular carcinoma cells harbors cancer stem cell-like properties. Hepatology. 2006;44(1):240-51. doi:10.1002/hep.21227.

10. Ma S, Tang KH, Chan YP, Lee TK, Kwan PS, Castilho A, et al. miR-130b Promotes CD133(+) liver tumor-initiating cell growth and self-renewal via tumor protein 53 -induced nuclear protein 1. Cell Stem Cell. 2010;7(6):694-707. doi:10.1016/j.stem.2010.11.010.

11. Yang ZF, Ho DW, Ng MN, Lau CK, Yu WC, Ngai P, et al. Significance of CD90+ cancer stem cells in human liver cancer. Cancer Cell. 2008:13(2):153-66. doi:10.1016/i.ccr.2008.01.013.

12. Yamashita $T$, Honda M, Nakamoto $Y$, Baba M, Nio K, Hara $Y$, et al. Discrete nature of EpCAM+ and CD90+ cancer stem cells in human hepatocellular carcinoma. Hepatology. 2013;57(4):1484-97. doi:10.1002/ hep.26168.

13. Rege TA, Hagood JS. Thy-1, a versatile modulator of signaling affecting cellular adhesion, proliferation, survival, and cytokine/growth factor responses. Biochim Biophys Acta. 2006;1763(10):991-9. doi:10.1016/ j.bbamcr.2006.08.008.

14. Herrera MB, Bruno S, Buttiglieri S, Tetta C, Gatti S, Deregibus MC, et al. Isolation and characterization of a stem cell population from adult human liver. Stem Cells. 2006;24(12):2840-50. doi:10.1634/stemcells.2006-0114.

15. Sukowati $C H$, Anfuso B, Torre G, Francalanci P, Croce LS, Tiribelli C. The expression of CD90/Thy-1 in hepatocellular carcinoma: an in vivo and in vitro study. PLoS One. 2013;8(10):e76830. doi:10.1371/journal.pone.0076830.

16. Lingala S, Cui YY, Chen X, Ruebner BH, Qian XF, Zern MA, et al. Immunohistochemical staining of cancer stem cell markers in hepatocellular carcinoma. Exp Mol Pathol. 2010;89(1):27-35. doi:10.1016/ j.yexmp.2010.05.005.

17. Yu XH, Xu LB, Liu C, Zhang R, Wang J. Clinicopathological characteristics of 20 cases of hepatocellular carcinoma with bile duct tumor thrombi. Dig Dis Sci. 2011;56(1):252-9. doi:10.1007/s10620-010-1256-8.

18. Lu JW, Chang JG, Yeh KT, Chen RM, Tsai JJ, Hu RM. Overexpression of Thy $1 /$ CD90 in human hepatocellular carcinoma is associated with HBV infection and poor prognosis. Acta Histochem. 2011;113(8):833-8. doi:10.1016/j.acthis.2011.01.001. 
19. Yang ZF, Ngai P, Ho DW, Yu WC, Ng MN, Lau CK, et al. Identification of local and circulating cancer stem cells in human liver cancer. Hepatology. 2008;47(3):919-28. doi:10.1002/hep.22082.

20. Guo Z, Li LQ, Jiang JH, Ou C, Zeng LX, Xiang BD. Cancer stem cell markers correlate with early recurrence and survival in hepatocellular carcinoma. World J Gastroenterol. 2014;20(8):2098-106. doi:10.3748/wjg.v20.i8.2098.

21. Fang $L$, Zhang HB, Li H, Fu Y, Yang GS. miR-548c-5p inhibits proliferation and migration and promotes apoptosis in CD90(+) HepG2 cells. Radiol Oncol. 2012;46(3):233-41. doi:10.2478/v10019-012-0025-z.

22. Fontana S, Saieva L, Taverna S, Alessandro R. Contribution of proteomics to understanding the role of tumor-derived exosomes in cancer progression: state of the art and new perspectives. Proteomics. 2013;13(10-11):1581-94. doi:10.1002/pmic.201200398.

23. Thery C, Amigorena S, Raposo G, Clayton A. Isolation and characterization of exosomes from cell culture supernatants and biological fluids. Current protocols in cell biology / editorial board, Juan S Bonifacino [et al]. 2006; Chapter 3:Unit 3 22. doi:10.1002/0471143030.cb0322s30.

24. Braicu C, Tomuleasa C, Monroig P, Cucuianu A, Berindan-Neagoe I, Calin GA. Exosomes as divine messengers: are they the Hermes of modern molecular oncology? Cell Death Differ. 2015;22(1):34-45. doi:10.1038/cdd.2014.130.

25. Kogure T, Yan IK, Lin WL, Patel T. Extracellular vesicle-mediated transfer of a novel long noncoding RNA TUC339: a mechanism of intercellular signaling in human hepatocellular cancer. Genes Cancer. 2013;4(7-8):261-72. doi:10.1177/1947601913499020

26. Taverna S, Flugy A, Saieva L, Kohn EC, Santoro A, Meraviglia S, et al. Role of exosomes released by chronic myelogenous leukemia cells in angiogenesis. Int J Cancer. 2012;130(9):2033-43. doi:10.1002/ijc.26217.

27. Corrado C, Raimondo S, Saieva L, Flugy AM, De Leo G, Alessandro R. Exosome-mediated crosstalk between chronic myelogenous leukemia cells and human bone marrow stromal cells triggers an interleukin 8-dependent survival of leukemia cells. Cancer Lett. 2014;348(1-2):71-6. doi:10.1016/j.canlet.2014.03.009.

28. Webber J, Yeung $\mathrm{V}$, Clayton A. Extracellular vesicles as modulators of the cancer microenvironment. Semin Cell Dev Biol. 2015;40:27-34. doi:10.1016/ j.semcdb.2015.01.013.

29. Eun JR, Jung YJ, Zhang Y, Zhang Y, Tschudy-Seney B, Ramsamooj R, et al. Hepatoma SK Hep-1 cells exhibit characteristics of oncogenic mesenchymal stem cells with highly metastatic capacity. PLoS One. 2014;9(10):e110744. doi:10.1371/journal.pone.0110744

30. Yu FJ, Zheng JJ, Dong PH, Fan XM. Long non-coding RNAs and hepatocellular carcinoma. Mol Clin Oncol. 2015;3(1):13-7. doi:10.3892/mco.2014.429.

31. Sun J, Bie B, Zhang S, Yang J, Li Z. Long Non-coding RNAs: critical players in hepatocellular carcinoma. Int J Mol Sci. 2014;15(11):20434-48. doi:10.3390/ijms151120434.

32. Pan YF, Qin T, Feng L, Yu ZJ. Expression profile of altered long non-coding RNAs in patients with HBV-associated hepatocellular carcinoma. J Huazhong Univ Sci Technol Med sci Hua zhong ke ji da xue xue bao Yi xue Ying De wen ban = Huazhong keji daxue xuebao Yixue Yingdewen ban 2013;33(1):96-101. doi:10.1007/s11596-013-1078-y.

33. Yang X, Xie X, Xiao YF, Xie R, Hu CJ, Tang B et al. The emergence of long non-coding RNAs in the tumorigenesis of hepatocellular carcinoma. Cancer letters. 2015. doi: 10.1016/j.canlet.2015.02.035

34. George J, Patel T. Noncoding RNA as therapeutic targets for hepatocellular carcinoma. Semin Liver Dis. 2015;35(1):63-74. doi:10.1055/s-0034-1397350.

35. Ma C, Nong K, Zhu H, Wang W, Huang X, Yuan Z, et al. H19 promotes pancreatic cancer metastasis by derepressing let-7's suppression on its target HMGA2-mediated EMT. Tumour biology. J Int Soc Oncodev Biol Med. 2014;35(9):9163-9. doi:10.1007/s13277-014-2185-5.

36. Matouk IJ, Raveh E, Abu-lail R, Mezan S, Gilon M, Gershtain E, et al. Oncofetal H19 RNA promotes tumor metastasis. Biochim Biophys Acta. 2014;1843(7):1414-26. doi:10.1016/j.bbamcr.2014.03.023.

37. Takahashi K, Yan IK, Kogure T, Haga H, Patel T. Extracellular vesicle-mediated transfer of long non-coding RNA ROR modulates chemosensitivity in human hepatocellular cancer. FEBS open Bio. 2014;4:458-67. doi:10.1016/ j.fob.2014.04.007

38. Ye J, Wu D, Wu P, Chen Z, Huang J. The cancer stem cell niche: cross talk between cancer stem cells and their microenvironment. Tumour biology. J Int Soc Oncodev Biol Med. 2014;35(5):3945-51. doi:10.1007/s13277-013-1561-x.

39. Kahlert C, Kalluri R. Exosomes in tumor microenvironment influence cancer progression and metastasis. J Mol Med (Berl). 2013;91(4):431-7. doi:10.1007/ s00109-013-1020-6.
40. Takahashi K, Yan IK, Haga H, Patel T. Modulation of hypoxia-signaling pathways by extracellular linc-RoR. J Cell Sci. 2014;127(Pt 7):1585-94. doi:10.1242/jcs.141069.

41. Moran VA, Perera RJ, Khalil AM. Emerging functional and mechanistic paradigms of mammalian long non-coding RNAs. Nucleic Acids Res. 2012;40(14):6391-400. doi:10.1093/nar/gks296.

42. Zhang Y, Tycko B. Monoallelic expression of the human H19 gene. Nat Genet. 1992;1(1):40-4. doi:10.1038/ng0492-40.

43. Hark AT, Schoenherr CJ, Katz DJ, Ingram RS, Levorse JM, Tilghman SM. CTCF mediates methylation-sensitive enhancer-blocking activity at the H19/lgf2 locus. Nature. 2000:405(6785):486-9. doi:10.1038/35013106.

44. Dey BK, Pfeifer K, Dutta A. The H19 long noncoding RNA gives rise to microRNAs miR-675-3p and miR-675-5p to promote skeletal muscle differentiation and regeneration. Genes Dev. 2014;28(5):491-501. doi:10.1101/gad.234419.113.

45. Kallen AN, Zhou XB, Xu J, Qiao C, Ma J, Yan L, et al. The imprinted H19 IncRNA antagonizes let-7 microRNAs. Mol Cell. 2013:52(1):101-12. doi:10.1016/j.molcel.2013.08.027.

46. Yang F, Bi J, Xue X, Zheng L, Zhi K, Hua J, et al. Up-regulated long non-coding RNA H19 contributes to proliferation of gastric cancer cells. FEBS J. 2012;279(17):3159-65. doi:10.1111/j.1742-4658.2012.08694.x.

47. Luo M, Li Z, Wang W, Zeng Y, Liu Z, Qiu J. Long non-coding RNA H19 increases bladder cancer metastasis by associating with EZH2 and inhibiting E-cadherin expression. Cancer Lett. 2013;333(2):213-21. doi:10.1016/j.canlet.2013.01.033.

48. Matouk IJ, DeGroot N, Mezan S, Ayesh S, Abu-lail R, Hochberg A, et al. The H19 non-coding RNA is essential for human tumor growth. PLoS One. 2007;2(9):e845. doi:10.1371/journal.pone.0000845

49. Fellig Y, Ariel I, Ohana P, Schachter P, Sinelnikov I, Birman T, et al. H19 expression in hepatic metastases from a range of human carcinomas. J Clin Pathol. 2005;58(10):1064-8. doi:10.1136/jcp.2004.023648.

50. Kim DK, Zhang L, Dzau VJ, Pratt RE. H19, a developmentally regulated gene, is reexpressed in rat vascular smooth muscle cells after injury. J Clin Invest. 1994;93(1):355-60. doi:10.1172/JCl116967.

51. Han DK, Khaing ZZ, Pollock RA, Haudenschild CC, Liau G. H19, a marker of developmental transition, is reexpressed in human atherosclerotic plaques and is regulated by the insulin family of growth factors in cultured rabbit smooth muscle cells. J Clin Invest. 1996;97(5):1276-85. doi:10.1172/JCI118543.

52. Noto R, Santangelo MG, Ricagno S, Mangione MR, Levantino M, Pezzullo M, et al. The tempered polymerization of human neuroserpin. PLoS One. 2012;7(3):e32444. doi:10.1371/journal.pone.0032444.

\section{Submit your next manuscript to BioMed Central and take full advantage of:}

- Convenient online submission

- Thorough peer review

- No space constraints or color figure charges

- Immediate publication on acceptance

- Inclusion in PubMed, CAS, Scopus and Google Scholar

- Research which is freely available for redistribution 